\title{
Image Denoising Based on Hybrid Fourier and Neighborhood Wavelet Coefficients
}

\author{
Jun Cheng, Songli Lei \\ College of Physical and Information Science, Hunan Normal University, Changsha, China \\ Hunan Art Professional College, Changsha, China \\ chengjun222@yahoo.com.cn
}

Keywords: image denoising; Fourier transform; neighboring wavelet coefficients; PSNR

\begin{abstract}
Image denoising is a classical topic and a difficult problem in the field of image processing. Fourier transform can effectively sparsely represent the smoothing texture part of the image, but can't effectively represent mutations in the image. The wavelet transform can sparsely represent sharply changing parts in the image, but can't effectively represent the texture and the slowly changing parts of the image. A new method based on hybrid Fourier and neighborhood wavelet coefficient is presented, and experiments show that the method is valid and can improve the visual effect, and that the objective indicator PSNR is better than NeightShrink method.
\end{abstract}

\section{Introduction}

The image is always affected by a variety of noise interference in the process of acquisition and transmission. These noises have very big impact on the image compression, feature extraction and edge detection. In order to improve the quality of the image post-processing, the image denoising pretreatment is necessary[1].

Theoretically, if only few coefficients of images have high-magnitude and most coefficients are close to zero, the performance of transform domain denoising methods would be good[2]. However, whether we can use sparse representation in the transform domain depends on the feature of the image and the type of the transform. Fourier transform can effectively sparsely represent the part of a certain conversion cycle with regular and smooth texture in the image, but can't effectively express mutations in the part of the image, such as image edges. Wavelet transform can sparsely represent the sharply changing section in the image, but can't effectively represent the texture and slowly changing parts in the image. This paper presents a hybrid Fourier-neighbor wavelet coefficients shrinkage image denoising method, and experiments show that the method can better protect the texture and slowly changing part of the image and can better restore the original image.

\section{Hybrid Fourier-Wavelet Transform Image Denoising Algorithm}

In the process of image denoising, the original image is inevitably distorted. In fact, image denoising makes trade-off between the noise suppression and the original image retention. Due to the non-stationary feature of the image, the wavelet transform is generally more suitable for image denoising. Therefore, in the hybrid Fourier-Wavelet transform image denoising algorithm, wavelet transform plays a dominant role and the Fourier transform plays a supporting role. The main steps are as follow:

1) Reduce the noise conservatively in the Fourier domain, which can reduce the level of noise without unduly distort the original image;

2) Remove the remaining noise in the wavelet domain.

For zero-mean Gaussian white noise, what will be filtered in wavelet domain the second step is actually colored noise after the first step. Therefore it is necessary to study the wavelet coefficient characteristics of the colored noise.

The Wavelet Coefficients of the Colored Noise. In general, the wavelet coefficients of the colored noise have the following two characteristics[3]: 
1) the wavelet coefficients correlation in each sub-band of the colored noise disappears rapidly;

2) the wavelet coefficients correlation between different sub-bands is small or almost irrelevant.

Because the wavelet transform is the approximate characteristics function of many mappings. The second feature can also be explained as follows: the mapping process of the signal from the time domain to the wavelet domain is equivalent to the signal through bandpass filtering. The wavelet coefficients in different subbands represent the signals of the different frequency bands, so the correlation of the wavelet coefficients between different subbands is weak. Experiments show that the wavelet coefficients of the colored noise in each subband is almost white noise, that the wavelet coefficients between the different subbands are uncorrelated, and that the variance of the noise is different indifferent sub-band.

Fourier Image Denoising. Consider a noisy image such as:

$$
y=x+n
$$

where $x$ is the original image, $n$ is zero mean Gaussian white noise, noise variance is $\delta^{2}$.

We use the wiener filter to reduce noise in Fourier domain. The wiener filter is a minimum mean squared error filter and requires prior knowledge of the power spectrum density of the original image and the power spectral density of the noise. Its transfer function is as the formula 2:

$$
H(w)=\frac{S(w)}{S(w)+N(w)}
$$

where $S(w)$ is the power spectral density of the original image, and $N(w)$ is the power spectral density of the noise. $Y(w)$ represents the Fourier transform of the noisy image. Assuming that the power spectral density of each frequency is equal to the power spectral of the adjacent frequency, then $S(w)$ can be estimated in a partial rectangular region $W(w)$ for every data point of $Y(w)$, as the formula 3:

$$
S(w)=\max \left(a, \frac{1}{M} \sum_{j \in W}|Y(j)|^{2}-\delta_{n}^{2}\right) \quad a>0
$$

where $M$ is the number of Fourier coefficients in a square window area $W(w), a$ is the smallest power spectral density of $x$.

In order not to distort the original image, we achieve the wiener filter conservatively as the following formula 4 :

$$
H(w)=\frac{b S(w)}{b S(w)+\delta_{n}^{2}} \quad b>1
$$

The result of the Fourier filtering is formula 5:

$z=x^{\prime}+n^{\prime}$

where $n^{\prime}$ is the remaining colored noise, $x^{\prime}$ is the slightly distorted image without noise.

$X^{\prime}(k)$ is used to denote the orthogonal wavelet coefficients of $X^{\prime}$, and the wavelet coefficients of $Z$ is shown in the following formula 6 :

$Z(k)=X^{\prime}(k)+N^{\prime}(k)$

where $N^{\prime}(k)$ is the wavelet coefficients of $n^{\prime}$. Although $N^{\prime}(k)$ is colored noise, the correlation of wavelet coefficients in each sub-band of the $N^{\prime}(k)$ disappears quickly, and the correlation between different sub-bands is very small or almost not correlated. Therefore the wavelet coefficients in each sub-band are almost white noise.

Neighborhood Wavelet Denoising. In the process of wavelet transform, low-pass and high-pass filter are recursively used for the low-frequency sub-band coefficients of the image. It means that the coefficient of the processed image is related in a small neighborhood [4]. This characteristic is named as layer correlation, and there may be a group of lager wavelet coefficients, and vice versa[5].

Let us assume that $d_{m, n}$ is wavelet transform coefficient of the noisy image, and define that 
$W_{m, n}(d)$ is neighborhood window, and its side length is $d$ and its center is the thresholding wavelet coefficient.

Shrink the thresholding wavelet coefficients by the formula 7 :

$d_{m, n}^{\prime}=d_{m, n} \alpha_{m, n}$

where $d_{m, n}^{\prime}$ is the thresholded value, the shrinkage factor is defined as in the formula 8 :

$$
\begin{aligned}
& \alpha_{m, n}= \begin{cases}1-\left(\frac{T}{S_{m, n}}\right)^{2} & S_{m, n}>T(T \text { is threshold value }) \\
0 & \text { other }\end{cases} \\
& S_{m, n}^{2}=\sum_{(m, n) \in W_{m, n}} d_{m, n}^{2}
\end{aligned}
$$

Threshold selection is the key of wavelet threshold denoising method, and the most classic threshold is common threshold proposed by Donoho [6]. The common threshold is defined by formula 10:

$$
T=\sigma \sqrt{2 \ln \left(N^{*} M\right)}
$$

where $\mathrm{N}$ and $\mathrm{M}$ are the image sizes, and $\sigma$ is the noise variance which need to be estimated in advance. Donoho also gives the following estimation formula:

$$
\hat{\sigma}=\frac{\text { Median }\left(\left|W_{i . j}\right|\right)}{0.6745}, W_{i j} \in \text { subbandHH }_{1}
$$

where $W_{i, j}$ denotes the wavelet coefficient matrix in the diagonal direction of the first layer (the highest resolution) image wavelet decomposition.

\section{Experiment and analysis}

In order to verify the effectiveness of the proposed method, we select some grayscale images to test. These images include Lena, Barbara, House. The image Barbara has more texture, Lena is a relatively smooth image, and House has sharp edges.

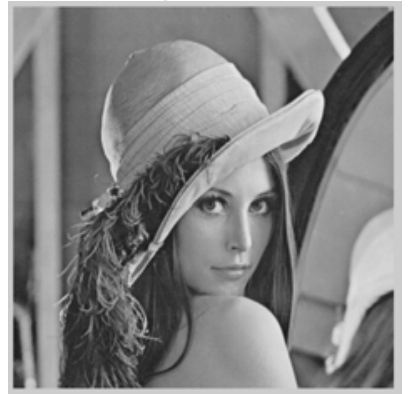

(a)lena

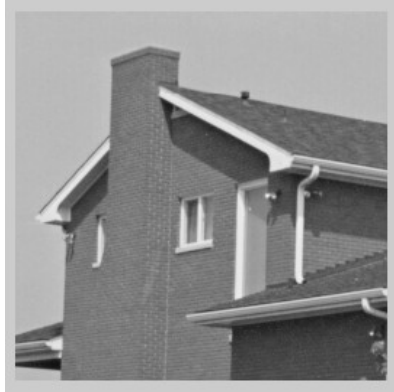

(b)house

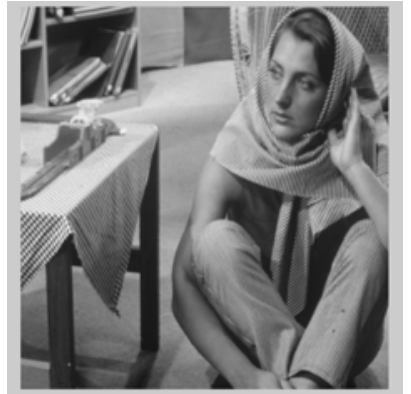

(c) barbara

Fig.1: the images used in the experiments

In all experiments, we use orthogonal wavelet transform and select $\mathrm{db} 8$ as wavelet basis, and wavelet decomposition level is four. We find that the noise reduction effect is good when a is $0.1 \delta^{2}{ }_{n}$, b is 5 , the window is $7 * 7$ in Fourier domain and the window is $3 * 3$ in wavelet domain. Make the denoising experiments in accordance with the following steps:

1) in the Fourier domain:

a) estimate power spectral density of the noisy image with formula 3 ;

b) reduce the noise with Wiener filter 4;

2) in the wavelet domain:

a) decompose the noisy image through wavelet transform to get wavelet coefficients in different scales $\mathrm{w}_{\mathrm{i}, \mathrm{j}}^{\mathrm{k}}$ and different sub-bands;

b) calculate the current decomposition scale threshold $\mathrm{T}_{\mathrm{k}}$ with the threshold estimation formula;

c) calculate the local energy of the current detail coefficients in the selected neighborhood and shrink the detail coefficients according to the formula to obtain the modified coefficients; 
d) repeat step (b) and (c) to get all the high-frequency sub-band detail coefficients

e) make the inverse wavelet transform with the processed wavelet coefficients to reconstruct the denoised image;

f) evaluate the quality of the filtering effect.

In order to evaluate objectively denoising effect of different methods, we select PSNR to measure denoising performance, and PSNR is defined as follows:

$$
\begin{aligned}
& M S E=\frac{\sum_{i=0}^{M-1} \sum_{j=0}^{N-1}(f(i, j)-\hat{f}(i, j))^{2}}{M * N} \\
& P S N R=10 \lg \left(\frac{255^{*} 255}{M S E}\right)
\end{aligned}
$$

Table 1 lists the PSNR value of two denoising methods with different noise strength.

Table 1: PSNR value comparison of different denoising

\begin{tabular}{|c|c|c|c|c|}
\hline$\sigma$ & 10 & 15 & 20 & 25 \\
\hline method & \multicolumn{5}{|c|}{ Lena $512 * 512$} \\
\hline NeighShrink & 31.8412 & 29.4906 & 27.0414 & 27.0223 \\
\hline proposed & 34.2568 & 32.5191 & 31.2265 & 30.2184 \\
\hline method & \multicolumn{5}{|c|}{ House $256 * 256$} \\
\hline NeighShrink & 32.7693 & 30.9773 & 29.6320 & 28.5646 \\
\hline proposed & 34.3020 & 32.3523 & 30.9769 & 29.9157 \\
\hline method & \multicolumn{5}{|c|}{ Barbara 512*512 } \\
\hline NeighShrink & 29.9304 & 27.6823 & 26.3258 & 25.0765 \\
\hline proposed & 31.4067 & 29.7338 & 28.4451 & 27.4188 \\
\hline
\end{tabular}

Usually, larger is the PSNR value, better is the denoising effect, and better is the quality of the reconstructed image. Compared to NeighShrink method, the PSNR value derived by the proposed method is significantly higher in various noise levels according to table1.

Figure 2 shows image denoising effects of the two methods when the noise standard deviation is equal to 25 .
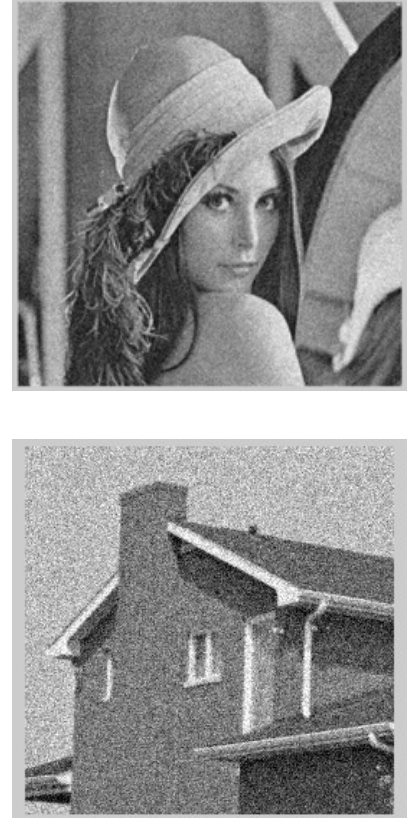

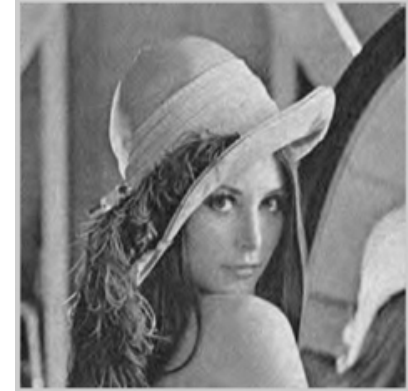

(a)

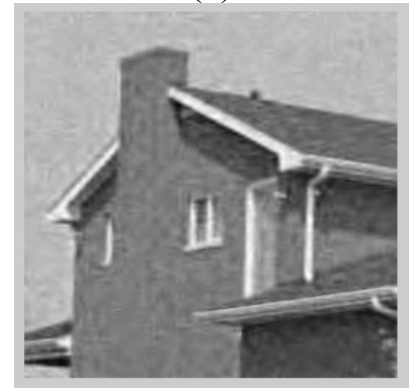

(b)
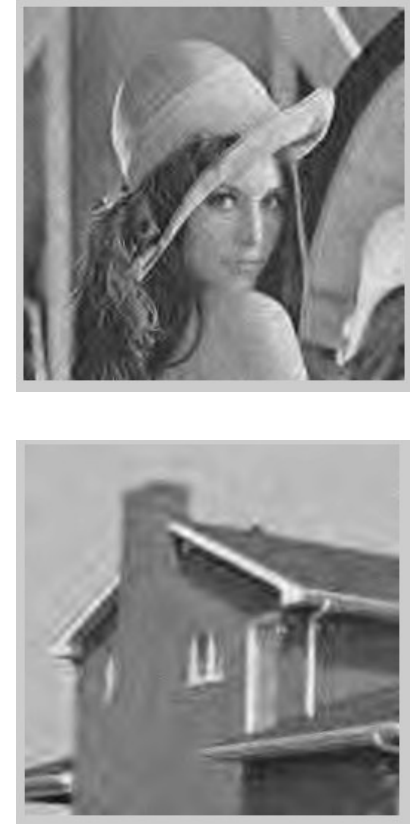

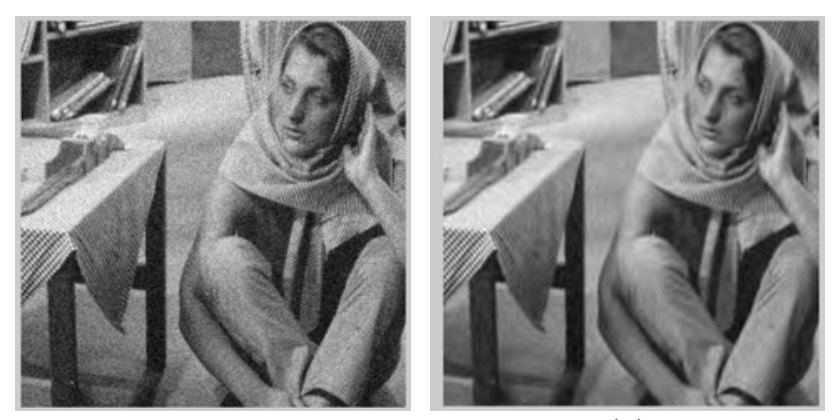

(c)

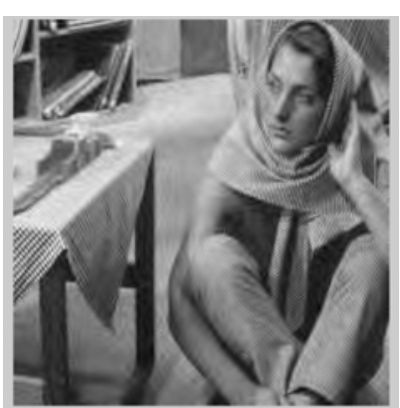

Fig. 2 image denoising effect when the noise standard deviation is equal to 25 (the left is noisy image, the middle is hybrid algorithm denoised image, and the right is NeighShrink algorithm denoised image)

(a) Lena (b) House (c) Barbara

Compared to NeighShrink method, the proposed method not only improves the blurring of the visual effects and retains the edges of the image and can better handle image texture.

\section{Conclusions}

This paper proposes a hybrid Fourier-wavelet transform denoising method. It can significantly improve the filtering effect, and more efficiently preserve image details. The experiment results show that this method not only obtains a significant improvement in visual, but also increases the value of PSNR significantly.

\section{Acknowledgment}

This project is supported by the Hunan Provincial Education Department under Grant $110528(11 \mathrm{C} 0801)$.

\section{References}

[1] Portilla J.,Strela V., Wainwright M J.,et al. Image denoising using scale mixtures of Gaussians in the wavelet domain. IEEE Trans. Image Processing, 2003,12(11):1338-1351

[2] Weaver J. Xu Y., Healy D., et al. Filtering MR images in the wavelet transform domain. Magn. Reson. Med, 1991,21:288-295

[3] Jiang S, HaoX. Hybrid Fourier-wavelet image denoising. Electron. Lett,2007,43(20)):1081-1082

[4] Donoho DL. Johnstone I M. Ideal spatial adaptation by wavelet shrinkage. Biometrika.1994,81

(3):425-455

[5] Chen G Y, Bui T D, Krzyzak A. Image denoising using neighbouring wavelet coefficients[J]. Integrated Computer-Aided Engineering, 2005, 12(1):99-107

[6] Donoho D L. Johnstone I M. Adapting to unknown smoothness via wavelet shrinkage. J. Amer. Stat. Assoc., 1995,90(432):1200-1224 